\title{
Kink shape solutions of the Maxwell-Lorentz system
}

\author{
Sørensen, Mads Peter; Webb, G.M.; Brio, M.; Moloney, J.V.
}

Published in:

Physical Review E. Statistical, Nonlinear, and Soft Matter Physics

Link to article, DOI:

10.1103/PhysRevE.71.036602

Publication date:

2005

Document Version

Publisher's PDF, also known as Version of record

Link back to DTU Orbit

Citation (APA):

Sørensen, M. P., Webb, G. M., Brio, M., \& Moloney, J. V. (2005). Kink shape solutions of the Maxwell-Lorentz system. Physical Review E. Statistical, Nonlinear, and Soft Matter Physics, 71(3), 036602.

https://doi.org/10.1103/PhysRevE.71.036602

\section{General rights}

Copyright and moral rights for the publications made accessible in the public portal are retained by the authors and/or other copyright owners and it is a condition of accessing publications that users recognise and abide by the legal requirements associated with these rights.

- Users may download and print one copy of any publication from the public portal for the purpose of private study or research.

- You may not further distribute the material or use it for any profit-making activity or commercial gain

- You may freely distribute the URL identifying the publication in the public portal 


\title{
Kink shape solutions of the Maxwell-Lorentz system
}

\author{
Mads Peter Sørensen* \\ Department of Mathematics, Technical University of Denmark, DK-2800 Kongens Lyngby, Denmark \\ Garry M. Webb \\ Institute of Geophysics and Planetary Physics, University of California Riverside, Riverside, California 92521, USA \\ Moysey Brio ${ }^{\ddagger}$ and Jerome V. Moloney ${ }^{\S}$ \\ Department of Mathematics, Arizona Center for Mathematical Sciences, University of Arizona, Tucson, Arizona 85721, USA
}

(Received 20 June 2003; revised manuscript received 3 November 2004; published 8 March 2005)

\begin{abstract}
In the limit of high amplitude oscillating electromagnetic fields, a sequence of kink antikink shaped optical waves has been found in the Maxwell's equations coupled to a single Lorentz oscillator and with Kerr nonlinearity. The individual kinks and antikinks result from a traveling wave assumption and their stability has been assessed by numerical simulations. For typical physical parameter values the kink width is of the order of tens of femtoseconds.
\end{abstract}

DOI: 10.1103/PhysRevE.71.036602

PACS number(s): 42.70.Jk, 42.65.Re, 42.65.Tg

\section{INTRODUCTION}

Optical pulse propagation in fibers is usually studied using the nonlinear Schrödinger (NLS) equation that is valid for slow envelope dynamics of the pulse [1]. However, for ultrashort pulses a first principles approach has been proposed that is based on the vector Maxwell's equations coupled to one or more Lorentz oscillators modeling the interaction between light and media [2,3]. The Lorentz oscillators provide dispersion. For high intensity pulses, the constitutive relation between the displacement current and the electric field includes a cubic Kerr nonlinearity.

Optical envelope pulses in the nonlinear Maxwell-Lorentz system can be described by the NLS equation or extended versions of the NLS equation taking into account higher order dispersion and nonlinearities. In Refs. [4,5] the NLS equation and extended versions of the NLS equation have been derived and a comparison between full numerical solutions of the Maxwell-Lorentz system and the NLS equation show remarkable agreement even for very short pulses with only 3-4 oscillation periods. In the literature not only have envelope pulses been studied but single humped localized pulses can be found in models of the Maxwell's equations coupled to linear as well as nonlinear oscillators [6-8]. These pulses are traveling solitary waves with solitonlike properties and are expected to be found in studies of atomic physics by means of photoionization leading to intense and nonoscillatory electromagnetic fields. Experimental observations of traveling waves in lasers have been reported in [9].

\footnotetext{
*Electronic address: M.P.Soerensen@mat.dtu.dk; URL: http:// www.mat.dtu.dk

${ }^{\dagger}$ Electronic address: gmwebb@citrus.ucr.edu; URL: http:// www.igpp.ucr.edu

‡Electronic address: brio@acms.arizona.edu

${ }^{\S}$ Electronic address: jml@acms.arizona.edu; URL: http:// acms.arizona.edu
}

For the Maxwell's equations coupled to one Lorentz oscillator, we show that the oscillating electromagnetic field converges toward a train of traveling kinks and antikinks with a period going toward infinity as the oscillation amplitude increases. The individual kinks rise from a plateau of negative constant electric field to a plateau of positive constant electric field of the same absolute value. The antikink decreases from a positive plateau to a negative plateau. The change of the electric field value takes place over a time interval of the order of tens of femtoseconds in strongly nonlinear materials as optical polymers $[10,11]$. These kink and antikink solutions have been found by a traveling wave assumption and their stability is verified by numerical simulations. Reference [12] investigates the dispersion equation of the Maxwell-Lorentz system and compares bright and solitary waves of the appropriate NLS equations, for the case of cubic and quintic Kerr nonlinearities. The latter investigation shows that blowup or collapse does not occur in the Maxwell-Lorentz system. However, for the case of quintic nonlinearity, the vector Maxwell system does exhibit an initial collapse similar to the quintic NLS equation, but the collapse is eventually arrested.

After describing the model (Sec. II) we present a phase plane analysis of the kink solution (Sec. II A) followed by numerical simulations of kink anti-kink pairs that verify their stability (Sec. III). Our summary is given in Sec. IV.

\section{MAXWELL-LORENTZ MODEL}

Consider the propagation of light in media with dispersion and nonlinearity by using Maxwell's equations. Assuming transverse plane waves propagating in the $z$-axis direction we introduce the electric field $\mathbf{E}=(E(z, t), 0,0)$, the magnetic field $\mathbf{B}=(0, B(z, t), 0)$, and the displacement current $\mathbf{D}$ $=(D(z, t), 0,0)$. The governing Maxwell's equations become

$$
\frac{\partial B}{\partial t}=-\frac{\partial E}{\partial z}, \quad \frac{\partial D}{\partial t}=-\frac{1}{\mu_{0}} \frac{\partial B}{\partial z}
$$

The parameter $\mu_{0}$ is the permeability of vacuum. Following the notation in Ref. [2], the displacement current is related to 
the electric field and the polarization through the constitutive equation

$$
D=\varepsilon_{0}\left[\varepsilon_{\infty} E+\Phi+a|E|^{2} E\right] .
$$

In the above equation $\Phi$ denotes the retarded or residual linear polarization response to an applied electric field and $\varepsilon_{\infty}$ is the infinite relative frequency permittivity arising from instantaneous polarization response. The nonlinear polarization is assumed to be an instantaneous cubic Kerr nonlinearity of strength $a$. The linear retarded response we shall model by a Lorentz oscillator, with resonance frequency $\omega_{0}$, coupled to the electric field [2]

$$
\frac{\partial^{2} \Phi}{\partial t^{2}}+\Gamma \frac{\partial \Phi}{\partial t}+\omega_{0}^{2} \Phi=\beta \omega_{0}^{2} E .
$$

The parameter $\beta$ is the difference between the static $\varepsilon_{s}$ and the infinite frequency relative permittivities $\beta=\varepsilon_{s}-\varepsilon_{\infty}$. The residual Raman molecular vibration response can be modeled using a similar linear oscillator but coupled to the field intensity $|E|^{2}$. However, in this study we neglect the Raman term. The term in $\Gamma$ describes a linear damping, which plays a key role in absorption experiments. However, in the limit of kink-antikink propagation the frequency goes toward zero and these nonlinear oscillations are far from resonance. The damping effect is to slowly deplete the waves as they propagate, a situation very much different from linear theory.

Noninstantaneous response results from inertia, i.e., the term $\Phi_{t t}$, in Eq. (3). Subscripts denote partial derivatives with respect to the subscript variable. In addition we can have nonlocal effects and wave guiding effects which for simplicity are not taken into account in this first approach. The Lorentz model describes a change of refractive index and it is added to the background index [see Eqs. (2) and (3)]. Therefore, negative relative permittivity is not possible. For numerical examples we shall use the representative physical values $[2,10,13]$

$$
\begin{gathered}
\varepsilon_{s}=5.25, \quad \varepsilon_{\infty}=2.25, \\
\omega_{0}=4.11 \times 10^{14} \mathrm{~Hz}, \quad a=2.7 \times 10^{-19} \frac{m^{2}}{V^{2}} .
\end{gathered}
$$

These parameter values correspond to electronic polarization in a highly nonlinear polymer. A resonance frequency of order $10^{14} \mathrm{~Hz}$ is typical for bounded electrons and the value stated for the third-order nonlinear parameter $a=\chi^{(3)}$ has been reported for a heterocyclic ladder polymer, nonether polyphenylquinoxaline [10]. Fused silica has a typical nonlinearity parameter of order $10^{-22} \mathrm{~m}^{2} / V^{2}[5]$.

\section{A. Traveling wave}

As the damping merely weakly depletes the oscillation in the off resonance case, we shall first neglect the influence of damping. Let us introduce the traveling wave assumption for the electric field $E(z, t)=E(\xi)$, where $\xi=z-v t$, and similarly for the magnetic field $B$, the displacement current $D$, and $\Phi$. Inserting this solution ansatz into Eqs. (1)-(3) we obtain the ordinary differential equation system

$$
\begin{gathered}
\frac{d E}{d \xi}=Y, \\
\frac{d Y}{d \xi}=\frac{6 a v^{2} E Y^{2}-\omega_{0}^{2}\left[\left(c^{2} / v^{2}-\varepsilon_{s}\right) E-a E^{3}\right]}{c^{2}-\varepsilon_{\infty} v^{2}-3 a v^{2} E^{2}} .
\end{gathered}
$$

In order to write the final equation for $E$ as a system of two first-order ordinary differential equations we have introduced $Y=E^{\prime}$, where the prime denotes differentiation with respect to $\xi$. For $v<c / \sqrt{\varepsilon_{s}}$ there exist three stationary solutions (fixed points) of the above system and they are

$$
(E, Y)=(0,0) \quad \text { and } \quad(E, Y)=\left( \pm \frac{1}{\sqrt{a}} \sqrt{\frac{c^{2}}{v^{2}}-\varepsilon_{s}}, 0\right) .
$$

In order to access the stability and the phase plane flow pattern around these three fixed points, we need to determine the eigenvalues of the Jacobian of the functions on the right hand sides of Eqs. (5) and (6) evaluated at the fixed points. In doing so we find the eigenvalues $\lambda$ of the Jacobian taken at $(E, Y)=(0,0)$ to be

$$
\lambda= \pm i \omega_{0} \sqrt{\frac{c^{2} / v^{2}-\varepsilon_{s}}{c^{2}-\varepsilon_{\infty} v^{2}}} .
$$

From this we learn that the stationary point $(E, Y)=(0,0)$ is a center and in the $(E, Y)$ phase plane we have elliptic circulating solution curves close to $(E, Y)=(0,0)$. These solutions are marginally stable. At the two other stationary points the Jacobian possesses identical eigenvalues, which are

$$
\lambda= \pm \sqrt{2} \omega_{0} \sqrt{\frac{c^{2} / v^{2}-\varepsilon_{s}}{\left(3 \varepsilon_{s}-\varepsilon_{\infty}\right) v^{2}-2 c^{2}}} .
$$

For the velocity lying in the interval

$$
c \sqrt{\frac{2}{3 \varepsilon_{s}-\varepsilon_{\infty}}}<|v|<c \frac{1}{\sqrt{\varepsilon_{s}}},
$$

we observe that the stationary points with nonvanishing values of $E$ in Eq. (7) are hyperbolic fixed points.

\section{NUMERICAL SIMULATION RESULTS}

In our numerical simulations we have rescaled the dependent and independent variables according to

$$
\begin{gathered}
z=k_{z} \widetilde{z}, \quad t=k_{t} \widetilde{t}, \quad \xi=k_{z} \widetilde{\xi}=k_{z}(\widetilde{z}-\widetilde{v} \widetilde{t}), v=\frac{k_{z}}{k_{t}} \widetilde{v}, \\
E(\xi)=k_{E} \widetilde{E}(\widetilde{\xi}),
\end{gathered}
$$

$$
\begin{gathered}
Y(\xi)=k_{Y} \tilde{Y}(\tilde{\xi}), \quad k_{t}=\frac{1}{\omega_{0}}, \quad k_{z}=\frac{c}{\omega_{0} \sqrt{\varepsilon_{\infty}}}, \quad k_{E}=\sqrt{\frac{\varepsilon_{\infty}}{3 a}}, \\
k_{Y}=\frac{\varepsilon_{\infty} \omega_{0}}{c \sqrt{3 a}} .
\end{gathered}
$$

The scaled and dimensionless variables are indicated by a tilde. In the scaled coordinates Eqs. (5) and (6) transform into 


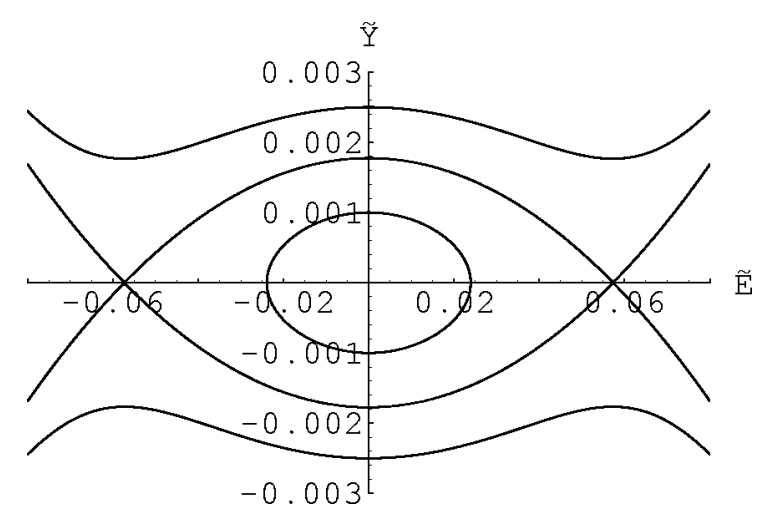

FIG. 1. Phase plane flow pattern calculated numerically from the scaled Eqs. (12), using the parameters in Eq. (4) and $\widetilde{v}$ $=0.6545$.

$$
\frac{d \tilde{E}}{d \tilde{\xi}}=\tilde{Y}, \quad \frac{d \tilde{Y}}{d \tilde{\xi}}=\frac{2 \widetilde{v}^{2} \widetilde{E} \tilde{Y}^{2}-\left(1 / \widetilde{v}^{2}-\varepsilon_{s} / \varepsilon_{\infty}\right) \tilde{E}+\widetilde{E}^{3} / 3}{1-\widetilde{v}^{2}-\widetilde{v}^{2} \widetilde{E}^{2}} .
$$

The phase plane flow pattern is shown in Fig. 1, obtained numerically by solving Eqs. (12). The scaled unstable fixed points are given by $\widetilde{E}=\sqrt{3\left[\left(1-\widetilde{v}^{2}\right) / \widetilde{v}^{2}-\beta / \varepsilon_{\infty}\right]}$. The upper heteroclinic trajectory connecting the left hyperbolic fixed point to the right hyperbolic fixed point forms the kinklike solution shown in Fig. 2 (solid curve). The antikink results from connecting the right hyperbolic fixed point with the left one along the lower heteroclinic curve. We stress that the kinklike solution is not an envelope for an oscillating carrier wave. Squaring the $E$ field gives a wave form which to some extent resembles a dark solitary wave. However, due to the absence of the carrier wave it is quite distinct from the usual dark soliton. The existence of the above kink shaped traveling wave solution does not imply that it is stable. In order to assess its stability, we have solved Eqs. (1)-(3) numerically using the scaled variables and by choosing initial conditions which are not exactly equal to the heteroclinic kink solution shown as the solid curve in Fig. 2. By choosing $\widetilde{E}(\widetilde{z}, 0)$ $=A_{\text {kink }} \tanh \left[\left(\widetilde{z}-z_{0}\right) / w\right]$ we start the simulations with an initial condition which is a fairly good approximation to the solu-

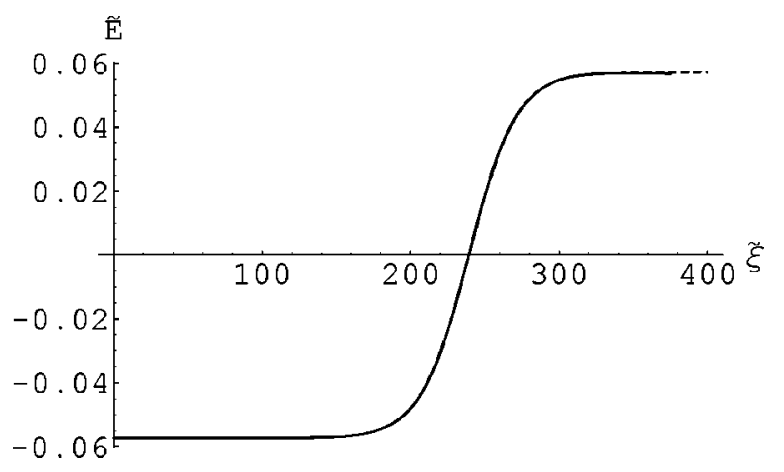

FIG. 2. Solid curve: The kink solution, $\widetilde{E}$ versus $\widetilde{\xi}$, calculated numerically from the scaled Eqs. (12). Dashed curve: The approximation $\widetilde{E}(\widetilde{\xi})=A_{\text {kink }} \tanh [(\widetilde{\xi}-239.2) / w], A_{\text {kink }}=5.7336 \times 10^{-2}$ and $w$ $=32.32$. The parameters are given in Eq. (4) and $\tilde{v}=0.6545$.
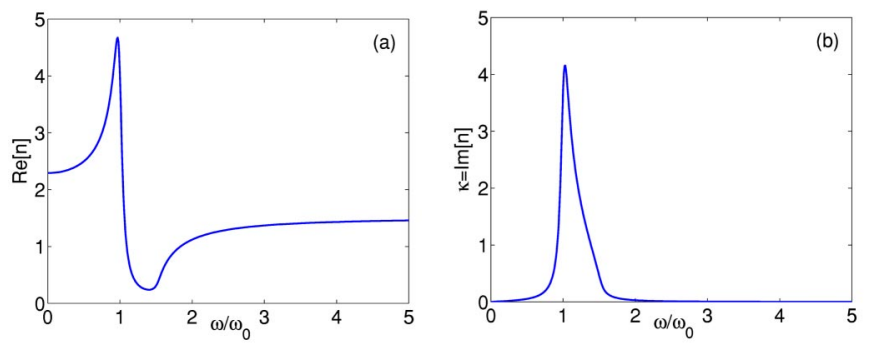

FIG. 3. The real part $\operatorname{Re}[n]$ (a) and the imaginary part $\kappa$ (b) of the index of refraction $n$ from Eq. (13) as function of the frequency ratio $\omega / \omega_{0}$. The normalized damping coefficient is $\gamma=0.1$.

tion, both near $\widetilde{\xi}=0$ and for $|\widetilde{\xi}| \rightarrow \infty$. The amplitude $A_{\text {kink }}$ and width $w$ are determined by matching the approximate tanhkink solution to the one obtained numerically from Eqs. (12). The comparison is shown in Fig. 2 where the dashed curve represents the tanh approximation.

In Fig. 1 the origin is a center point and nearby closed trajectories are elliptic curves corresponding to linear oscillating solutions. The dispersion relation for the linearized Eqs. (1)-(3), including the damping $\Gamma$, lead to a refractive index $n$ given by [14]

$$
n^{2}=c^{2} \frac{k^{2}}{\omega^{2}}=\varepsilon_{\infty}+\frac{\beta}{1-\left(\omega / \omega_{0}\right)^{2}-i \gamma \omega / \omega_{0}} .
$$

Here $k$ is the wave number of the plane wave ansatz $E$ $=E_{0} e^{i(k x-\omega t)}$, with $\omega$ being the frequency of $E$. In the above expression $\gamma=\Gamma / \omega_{0}$ is a normalized damping. The real part of the index of refraction, $\operatorname{Re}[n]$, is shown as function of $\omega / \omega_{0}$ in Fig. 3(a). In Fig. 3(b) we show the imaginary part $\kappa=\operatorname{Im}[n]$ of $n$ as function of $\omega / \omega_{0}$, which is the absorption line. Rather arbitrarily we have chosen $\gamma=0.1$. For vanishing damping the spectrum will show a forbidden gap in the frequency interval corresponding to negative slope of the real part of $n$ in Fig. 3(a).

We can now investigate the transition from the above linearized case to the nonlinear case by solving Eqs. (12) numerically. The results are shown in Fig. 4. Using $\tilde{v}=0.6545$, the initial conditions $(\tilde{E}(0), \tilde{Y}(0))=\left(0, \widetilde{Y}_{0}\right)$, and increasing $\tilde{Y}_{0}$, the oscillating solutions progressively become more nonlinear and hence different from the picture in Fig. 3. The oscillation period increases and the frequency decreases. The frequencies of the oscillations in the three figures are $\omega_{a} / \omega_{0}$ $=0.930, \omega_{b} / \omega_{0}=0.571$, and $\omega_{c} / \omega_{0}=0.307$, respectively, and the resonance frequency of the linearized system is $\omega_{0}$ $=2.87 \times 10^{-2}$. Comparing with Fig. 3(a) it is observed that as the oscillation amplitude increases, the frequency moves away from the resonance case close to $\omega_{0}$ leading to a substantial decrease of the absorption. Eventually, the solution turns into a form close to a train of kink-antikink nonlinear waves. In the phase plane plot this is a solution path following closely the heteroclinic orbits within their closure. This train of nonlinear solitary waves should be easy to obtain by increasing the amplitude of an external driving electromagnetic field. 

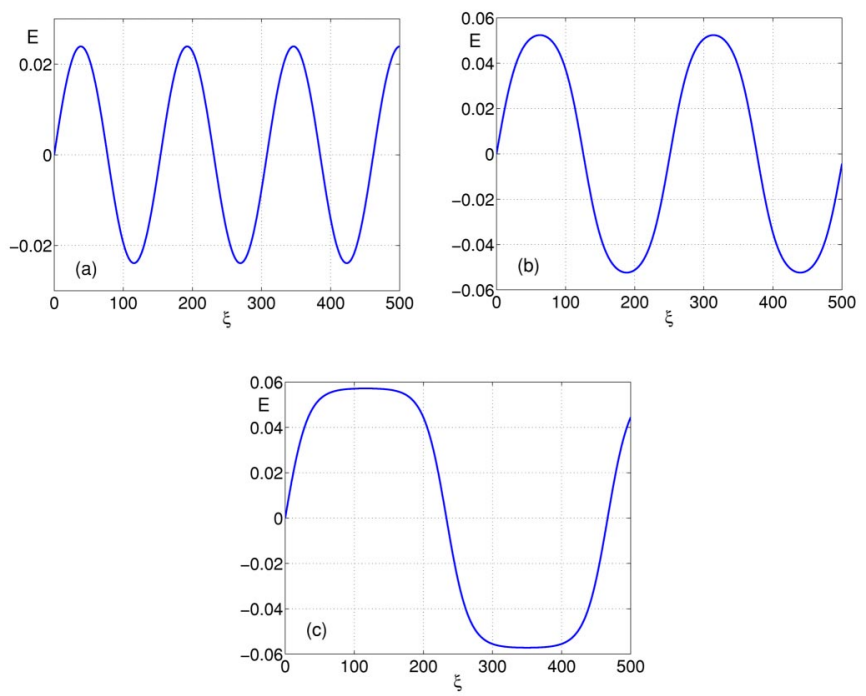

FIG. 4. Numerical solutions of Eqs. (12). (a) Oscillating waves corresponding to the nearly linear case starting from $(\tilde{E}(0), \tilde{Y}(0))$ $=(0,0.001)$. (b) Initial conditions $(\widetilde{E}(0), \tilde{Y}(0))=(0,0.00175)$. (c) A solution close to a train of kink-antikinks using the initial conditions $(\tilde{E}(0), \tilde{Y}(0))=(0,0.00177436) . \widetilde{v}=0.6545 . E$ and $t$ in the figure labels refer to the scaled variables $\widetilde{E}$ and $\tilde{t}$.

Figure 5 shows a kink and antikink pair traveling in the same direction. The reason for presenting a kink-antikink pair is due to our use of periodic boundary conditions in the numerical scheme, thereby allowing for simulating over long time stretches in a small spatial area without using adaptive grid generation. Only pairs of kinks and antikinks can satisfy these boundary conditions. Other types of boundary conditions could easily be imposed to handle single kinks but it will require more extensive simulations. Initially the kinks adjust their shape to the exact solution by shedding off linear radiation waves. After a transient period the kinks attain the shape given by the heteroclinic curves in Fig. 1. Our numerical simulations indicate that the kink shaped optical pulse is stable.

In normalized units the kink rises from $\widetilde{E}=-5.7336$ $\times 10^{-2}$ (in physical units $-9.556 \times 10^{7} \mathrm{~V} / \mathrm{m}$ ) to $\widetilde{E}$ $=+5.7336 \times 10^{-2}$ within a space interval of length about 59.3, taking as the full width at half maximum (FWHM) of

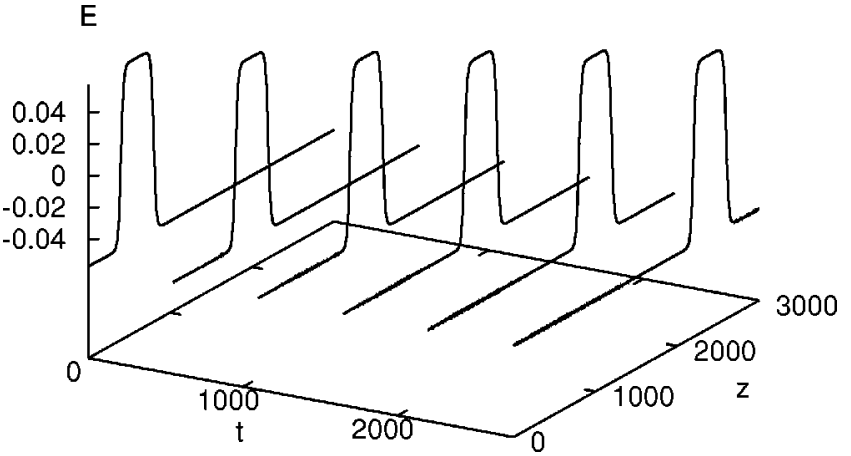

FIG. 5. A traveling kink and antikink wave, forming a squarelike pulse, using the parameter values in Eq. (4). The initial data for the kink are $\widetilde{E}(\widetilde{z}, 0)=A_{\text {kink }} \tanh \left[\left(\widetilde{z}-z_{1}\right) / w\right]$ where $A_{\text {kink }}=5.7336$ $\times 10^{-2}, w=32.32, \widetilde{v}=0.6545$, and $z_{1}=400$. For the antikink we used $A_{\text {kink }}=-5.7336 \times 10^{-2}, w=32.32, \widetilde{v}=0.6545$, and $z_{1}=800 . E$, $z$, and $t$ in the figure labels refer to the scaled variables $\widetilde{E}, \tilde{z}$, and $\tilde{t}$.

$\tilde{Y}$, the $\widetilde{\xi}$ derivative of the electric field. From Eq. (11) this corresponds to a physical distance of $28.8 \mu \mathrm{m}$. The speed of the traveling kink wave is $\widetilde{v}=0.6545$ and the associated physical wave speed becomes $v=\left(c / \sqrt{\varepsilon_{\infty}}\right) \tilde{v}=1.31 \times 10^{8} \mathrm{~m} / \mathrm{s}$ [see Eq. (11)]. Using the parameter values in Eq. (4), the change in the kink profile takes place within a time interval of about $220 \mathrm{fs}$, measured as the FWHM for the spatial derivative of the electric field (see Table I).

In Table I we present two other cases with different nonlinearity parameter $a$ and plateau values of the electric field $E$. The two first lines present results for a nonlinearity parameter $a$ representative of a highly nonlinear polymer $[10,13]$. The third line corresponds to fused silica. The width of the kink decreases as the electric field increases and the nonlinearity increases, which is to be expected.

\section{SUMMARY}

The vector Maxwell equations coupled to a single Lorentz oscillator with instantaneous Kerr nonlinearity possess a kink shaped traveling wave solution. Numerical simulations of initial conditions perturbed from the exact solution indicate that the kink solution is stable. Starting from linear oscillating plane waves and increasing the amplitude, the solution

TABLE I. The full width at half maximum of $\tilde{Y}$ for kinks in materials with different nonlinearity parameters $a$ and for different electric field strengths $E$ (plateau value). The physical kink velocity is denoted $v$ and the normalized velocity is denoted $\widetilde{v}$. The value of the nonlinearity parameter $a$ in the first two lines is representative for a highly nonlinear polymer. In the third line the $a$ value is representative for fused silica.

\begin{tabular}{cccccc}
\hline \hline $\begin{array}{c}a \\
\left(\mathrm{~m}^{2} / \mathrm{V}^{2}\right)\end{array}$ & $\begin{array}{c}v \\
(\mathrm{~m} / \mathrm{s})\end{array}$ & $\begin{array}{c}\tilde{v} \text { (normalized) } \\
\mathrm{F}\end{array}$ & $\begin{array}{c}E \\
(\mathrm{~V} / \mathrm{m})\end{array}$ & $\begin{array}{c}\text { (normalized) } \\
\mathrm{F}\end{array}$ & $\begin{array}{c}\text { FWHM } \\
(\mathrm{fs})\end{array}$ \\
\hline $2.7 \times 10^{-19}$ & $1.31 \times 10^{8}$ & 0.6545 & $9.56 \times 10^{7}$ & $5.73 \times 10^{-2}$ & 220 \\
$2.7 \times 10^{-19}$ & $1.20 \times 10^{8}$ & 0.60 & $1.92 \times 10^{9}$ & 1.15 & 15.2 \\
$1.03 \times 10^{-22}$ & $1.30 \times 10^{8}$ & 0.65 & $2.71 \times 10^{10}$ & 0.317 & 38.6 \\
\hline \hline
\end{tabular}


will become close to a train of kinks and antikinks with a frequency converging toward zero. This approach indicates that it should be experimentally easy to obtain the sequence of wave patterns close to kinks and antikinks. From known material parameters of silica and polymers $[10,13]$, we point to the possible physical realization of such a train, so far without taken into account wave guiding effects, spatially nonlocal effects, and Raman scattering. The kink solution in its nature is related to nonlinear localized optical pulses as described in Refs. [6-8].

\section{ACKNOWLEDGMENTS}

M.P.S. wishes to thank Arizona Center for Mathematical Sciences for their hospitality during completion of this work and acknowledges financial support from the Danish Technical Research Council through Project No. 21-02-0500. The work of G.M.W. was supported in part by NASA Grant No. NAG5-13451. The work of M.B. was partially supported by NSF Grants No. INT-0226872 and No. DMS 0335101 and AFOSR Grant No. f49620-03-1-0194.
[1] R. W. Boyd, Nonlinear Optics (Academic, New York, 1991).

[2] L. Gilles, S. C. Hagness, and L. Vázquez, J. Comput. Phys. 161, 379 (2000).

[3] G. Webb, M. P. Sørensen, M. Brio, A. R. Zakharian, and J. V. Moloney, Physica D 191, 49 (2004).

[4] C. V. Hile, Wave Motion 24, 1 (1996).

[5] C. V. Hile and W. L. Kath, J. Opt. Soc. Am. B 13, 1135 (1996).

[6] A. E. Kaplan and P. L. Shkolnikov, Phys. Rev. Lett. 75, 2316 (1995).

[7] A. I. Maimistov, in Nonlinearity and Disorder: Theory and Applications, edited by F. Kh. Abdullaev, O. Bang, and M. P. Sørensen (Kluwer, Dordrecht, 2002), pp. 263-278.
[8] E. V. Kazantseva, A. I. Maimistov, and B. A. Malomed, Opt. Commun. 188, 195 (2001).

[9] J. Houlihan, J. R. O'Callaghan, V. Voignier, G. Huyet, and J. G. McInerney, Opt. Lett. 26, 1556 (2001).

[10] J. Yan, J. Wu, H. Zhu, X. Zhang, D. Sun, F. Li, and M. Sun, Opt. Commun. 116, 425 (1995).

[11] B. A. Reinhardt, Trends Polym. Sci. 4, 287 (1996).

[12] M. P. Sørensen, M. Brio, G. M. Webb, and J. V. Moloney, Physica D 170, 287 (2002).

[13] P. Günter, Nonlinear Optical Effects and Materials, Springer Series in Optical Sciences Vol. 72 (Springer, Berlin, 2000).

[14] G. R. Fowles, Introduction to Modern Optics (Dover, New York, 1975). 